\title{
PROGRAMA ACADEMIA DA SAÚDE: OPERACIONALIDADE, AÇÕES E INTEGRAÇÃO
}

\author{
Health Gym Program: Operationality, actions and integration \\ Programa gimnasio de la salud: Operacionalización, acciones e integración
}

\author{
Laís Barreto de Brito Gonçalves (iD \\ Universidade Regional do Cariri - URCA - Crato (CE) - Brasil
}

Rachel Cardoso de Almeida (iD

Universidade Regional do Cariri - URCA - Crato (CE) - Brasil

Tainá Maranhão de Oliveira (iD

Universidade Regional do Cariri - URCA - Crato (CE) - Brasil

Maria Augusta Vasconcelos Palácio (iD

Universidade Federal do Vale do São Francisco - UNIVASF - Paulo Afonso (BA) -Brasil

Antônio Germane Alves Pinto (iD)

Universidade Regional do Cariri - URCA - Crato (CE) - Brasil

\section{RESUMO}

Objetivo: Analisar a percepção de usuários, gestores e trabalhadores da saúde sobre a operacionalidade, as ações desenvolvidas nos polos da Academia da Saúde e a sua integração com os demais pontos da Rede de Atenção à Saúde (RAS). Métodos: Estudo qualitativo desenvolvido em três municípios localizados na Macrorregião do Cariri, estado do Ceará, Brasil. Realizaramse entrevistas semi-estruturadas com os participantes do estudo: 15 usuários, três trabalhadores da saúde e três gestores envolvidos na execução, monitoramento e supervisão das atividades dos polos, entre outubro de 2016 e janeiro de 2017 . A análise dos dados foi pautada na proposição metodológica e analítica fundamentada na hermenêutica crítica. Resultados: $O$ Programa Academia da Saúde (PAS) tem demonstrado influências positivas na prática de atividade física da população usuária e, consequentemente, melhorias na condição de saúde e na qualidade de vida. Os resultados encontrados revelam a dinâmica de funcionamento e organização das atividades, destacando: as facilidades e dificuldades quanto a sua operacionalidade; as ações frequentemente desenvolvidas; os avanços observados em relação à promoção da saúde e à qualidade de vida; e a integralidade no PAS. Conclusão: Os usuários, os trabalhadores de saúde e os gestores compartilham a mesma percepção em relação à melhoria nas condições de saúde da população atendida nos polos da Academia da Saúde, e, no que se refere ao funcionamento, tem sido considerado bom, apesar de dificuldades relacionadas aos aspectos estruturais, de materiais e insumos dos polos em cada contexto estudado.

Descritores: Programas de Saúde; Promoção da Saúde; Integralidade em Saúde.

\section{ABSTRACT}

Objective: To analyze users', managers' and health workers' perception of the operationality of and actions developed in Health Gym facilities and their integration with other facilities within the Healthcare Network (Rede de Atenção à Saúde - RAS). Methods: This qualitative study was carried out in three municipalities located in the Macroregion of Cariri, Ceará State, Brazil. Semi-structured interviews were held with the study participants: 15 users, three health workers and three managers involved in the implementation, monitoring and supervision of the activities held in the facilities between October 2016 and January 2017. Data analysis was based on the methodological and analytical framework based on critical hermeneutics. Results: The Health Gym Program (Programa Academia da Saúde - PAS) has had a positive influence on engagement in physical activity among users and hence led to improvements in health status and quality of life. The findings show the dynamics of the operation and organization of the activities, particularly the advantages and difficulties regarding their operationality, the actions frequently developed, the observed advances in relation to health promotion and quality of life, and the comprehensiveness of the PAS. Conclusion: Users, health workers and managers share the same perception about the improvements in the health status of

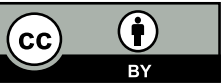


the population served by Health Gym facilities. Moreover, its operation is considered good despite the difficulties related to the structural, material and input aspects of the facilities in each context analyzed.

Descriptors: Health Programs; Health Promotion; Integrality in Health.

\section{RESUMEN}

Objetivo: Analizar la percepción de los usuarios, los gestores y los trabajadores sanitarios sobre la operacionalización, las acciones desarrolladas en los polos del Gimnasio de la Salud y su integración con los demás puntos de la Red de Atención a la Salud (RAS). Métodos: Estudio cualitativo desarrollado en tres municipios de la macro región de Cariri, estado de Ceará, Brasil. Se realizaron entrevistas semiestructuradas con los participantes del estudio: 15 usuarios, tres trabajadores sanitarios y tres gestores involucrados con la ejecución, el monitoreo y la supervisión de las actividades en los polos entre octubre de 2016 y enero de 2017. El análisis de los datos ha sido fundamentado en la proposición metodológica y analítica basada en la hermenéutica crítica. Resultados: El Programa Gimnasio de la Salud (PGS) ha presentado influencias positivas para la práctica de actividad física de la población usuaria y, en consecuencia, para la mejoría de la condición de salud y la calidad de vida. Los resultados revelan la dinámica de funcionamiento y organización de las actividades con realce para las facilidades y dificultades de su operacionalización; las acciones desarrolladas con frecuencia; los avances observados en la promoción de la salud y la calidad de vida; y la integralidad del PGS. Conclusión: Los usuarios, los trabajadores sanitarios y los gestores comparten la misma percepción respecto la mejoría de las condiciones de salud de la población asistida en los polos del Gimnasio de la Salud $y$, sobre el funcionamiento, lo mismo ha sido considerado bueno pese las dificultades de aspectos estructurales, materiales $y$ insumos de los polos en cada uno de los contextos estudiados.

Descriptores: Planes y Programas de Salud; Promoción de la Salud; Integralidad en Salud.

\section{INTRODUÇÃO}

O Programa Academia da Saúde (PAS) é uma estratégia de promoção da saúde e produção do cuidado para os municípios brasileiros, lançado em 2011 por meio da Portaria Ministerial $\mathrm{n}^{\circ} 1.401$, de 15 de junho, e redefinido pela Portaria $\mathrm{n}^{\circ} 2.681$, de 7 de novembro de 2013. A finalidade do PAS é implantar polos de academia com infraestruturas adequadas e profissionais qualificados para a prática e incentivo de atividades físicas, bem como estímulo a adoção de uma alimentação saudável nos municípios brasileiros, com custeio de recurso federal ${ }^{(1)}$. Objetivou-se, com a sua criação, promover práticas corporais, atividade física regular, alimentação saudável, educação em saúde, acompanhamento interdisciplinar, além de contribuir para a produção do cuidado de modos de vida saudáveis e sustentáveis na população em geral ${ }^{(2)}$.

O PAS oferece serviços que garantem cuidados voltados às particularidades do cenário atual, de adoecimento e mortalidade da população, sobretudo no que diz respeito às doenças crônicas não transmissíveis (DCNT). O mesmo configura-se como um ponto da Rede de Atenção à Saúde (RAS), potencializando ações e cuidados coletivos, e estabelecendo espaços apropriados para vivências e troca de conhecimentos ${ }^{(2)}$. O desenvolvimento do programa representa uma estratégia de políticas setoriais para melhoria da qualidade de vida da população por meio da Atenção Primária a Saúde (APS), de práticas de promoção à saúde, da integralidade das ações, do combate às DCNT e promoção de um estilo de vida saudável(3).

A implantação do PAS é resultado das repercussões trazidas pelo processo de industrialização e urbanização no país, que conduziram ao surgimento de grandes mudanças no padrão de morbimortalidade, o que trouxe um crescimento significativo das DCNT, responsáveis por mais da metade da mortalidade geral e pelos altos custos com internações, gerando grande impacto econômico para o sistema de saúde, além de uma diminuição na qualidade de vida e saúde da população( ${ }^{(4)}$. Estudos recentes têm mostrado que um terço dos adultos em todo o mundo são fisicamente inativos, causando mais de 5,3 milhões de mortes anualmente ${ }^{(5)}$, e essa inatividade física representa importante fator de risco para as DCNT.

Diante da necessidade de traçar metas que visem à diminuição desses índices, o Ministério da Saúde lançou, em 2011, o Plano Nacional para o Enfrentamento das DCNT ${ }^{(6)}$, que estabelece ações de promoção da saúde com o intuito de promover a realização de atividades físicas, a alimentação saudável, o envelhecimento ativo e a prevenção e controle do consumo excessivo de álcool e tabaco, mediante parcerias com a APS e trabalho multiprofissional e interdisciplinar, objetivos que se encaixam na ideia prioritária de criação do PAS ${ }^{(7)}$.

Com base na importância de se instalar polos do Academia da Saúde pelos diversos municípios brasileiros, no seu cenário nacional de implantação até maio do ano de 2015, constava um total de 1.165 polos de academia concluídos. Considerando os polos de origem similares, como o Programa Academia da Cidade (PAC), em Recife, 
Pernambuco, Brasil, esse total chegou a 4.240. Esses polos receberam o custeio federal para seu aperfeiçoamento, além de obras nos diversos estágios de construção, distribuídos por 2.849 municípios do país ${ }^{(8)}$.

O PAS surgiu a partir de experiências pontuais de incentivo à atividade física e, consequentemente, mudança de hábitos de vida, a partir de programas implementados em capitais brasileiras, como Recife, Aracaju, Belo Horizonte e Curitiba $^{(9)}$. Para o bom funcionamento do programa, os polos devem possuir infraestrutura e equipamentos adequados à prática de atividades individuais e coletivas, com orientação de profissionais capacitados ${ }^{(9)}$ que desenvolvam ações de promoção de atividade física e promoção da saúde direcionadas às especificidades de cada grupo. Além disso, essas ações devem estar vinculadas aos profissionais da APS.

As ações desenvolvidas pelo PAS têm como eixo transversal a promoção da saúde, a qual é entendida como a variação de opções que são oferecidas no intuito de promover a atuação ativa e autônoma do indivíduo sobre os fatores condicionantes e determinantes que afetam a saúde e qualidade de vida individual e coletiva, de modo que possam participar no controle desse processo ${ }^{(10)}$. A Política Nacional de Promoção da Saúde (PNPS) inclui ações que estão alinhadas com o PAS, como a alimentação saudável, prática de atividade física regular, prevenção e controle do tabagismo, além da redução do uso abusivo de álcool e outras drogas ${ }^{(10,11)}$.

Nesse sentido, a proposta de trabalho do PAS representa uma estratégia de promoção da saúde e que requer ações intersetoriais efetivas para a sua concretização. Para que a promoção da saúde seja consolidada, deve haver articulação entre os diversos setores da sociedade e com a população para a efetivação de ações intersetoriais, objetivando alcançar os propósitos da política de promoção da saúde ${ }^{(12)}$. A efetivação de ações intersetoriais implica o alcance da integralidade no PAS, uma vez que este precisa estar alinhado com os outros pontos de atenção da RAS, como as equipes de Saúde da Família e o Núcleo de Apoio à Saúde da Família (NASF), bem como, articulado com os diferentes atores que compõem esse processo, como gestores, trabalhadores da saúde e usuários.

Ademais, as ações políticas de promoção da saúde devem estar voltadas para as particularidades do ser individual e coletivo, com o intuito de garantir a autonomia e o autocuidado, em consonância com a articulação de estratégias entre as Redes de Atenção à Saúde ${ }^{(13)}$. A construção participativa entre usuários, gestores e trabalhadores da saúde proporciona o conhecimento das reais necessidades de cada comunidade, auxilia a traçar metas e estratégias a serem desenvolvidas nos polos, além de permitir observar a efetividade dos planos de cuidados, a articulação entre os envolvidos e o impacto causado na vida da população.

No Brasil, programas similares ao PAS, criados anteriormente por meio de iniciativas municipais de melhoria da saúde da população, promoviam ações de promoção de práticas de atividade física e promoção da saúde. A partir da criação do PAS, tiveram a oportunidade de candidatar-se ao custeio de suas ações, aperfeiçoando as estratégias já desenvolvidas previamente ${ }^{(4,14-17)}$.

Embora o programa delimite bem os objetivos e prioridades, estudos realizados com programas similares apontam limitações e desafios no funcionamento dos polos devido à falta de materiais, de infraestrutura e da formação adequada de profissionais, o que traz um impacto negativo na operacionalidade do mesmo e, desse modo, um declínio nos resultados esperados ${ }^{(3,17,18)}$.

Fatores facilitadores também estão presentes na realidade dos polos do PAS, como o aumento do vínculo que se estabelece entre a população e os profissionais envolvidos. Essa vinculação auxilia no conhecimento das reais necessidades da comunidade e de cada usuário, permitindo a elaboração de um plano de cuidados específicos para cada contexto, respeitando a subjetividade. Outro elemento positivo nesse processo é a intersetorialidade entre gestores e profissionais, que proporciona uma operacionalidade eficaz e ainda facilita a resolução de possíveis dificuldades e limitações ${ }^{(17)}$.

Desse modo, o estudo sobre o cotidiano dos polos do Academia da Saúde permite conhecer as atividades de promoção da saúde desenvolvidas, seu impacto na qualidade de vida e saúde da população participante do programa, além de identificar pontos positivos e negativos que influenciam a sua operacionalidade. Possibilita, ainda, avaliar a existência ou não de articulação entre profissionais ligados diretamente ao programa, gestores, profissionais da ESF e demais pontos da RAS.

Ancorados nessa discussão e diante do desejo de contribuir com o fortalecimento do PAS, parte-se do seguinte questionamento: "Como usuários, gestores e trabalhadores da saúde percebem o PAS a partir de questões relacionadas à operacionalidade e ações desenvolvidas nos polos do Programa e a sua integração com os demais programas ou pontos da RAS?" Para o melhor entendimento do objeto de estudo, utilizou-se a hermenêutica crítica, na qual o fundamento de análise é a práxis social na perspectiva crítico-analítica(19).

O objetivo desse estudo é analisar a percepção de usuários, gestores e trabalhadores da saúde sobre a operacionalidade, as ações desenvolvidas nos polos da Academia da Saúde e a sua integração com os demais pontos da Rede de Atenção à Saúde (RAS). 


\section{MÉTODOS}

Em adequação à natureza do objeto de estudo, optou-se por uma pesquisa qualitativa, a qual consiste na análise dos processos intersubjetivos do contexto social, ampliando-se o entendimento da produção humana a partir da hermenêutica crítica ${ }^{(19)}$. Nesse sentido, enfoca-se a compreensão das práticas de cuidado e gestão na operacionalidade cotidiana do Programa Academia da Saúde, situado na Macrorregião de saúde do Cariri cearense.

O estado do Ceará, Brasil, está dividido em cinco Macrorregiões de saúde (Fortaleza, Cariri, Sobral, Sertão Central e Litoral Leste), sendo cada uma subdividida em regiões, que totalizam 22 áreas que acomodam os municípios inerentes a cada uma ${ }^{(20)}$.

Desse modo, o estudo foi realizado em três municípios localizados na Macrorregião de saúde do Cariri, que mantêm polos do Programa Academia da Saúde e apresentam condições demográficas de médio e pequeno porte. A seleção ocorreu pela identificação dos representantes de cada grupo que integra o polo do Programa Academia da Saúde: usuários, profissionais de saúde e gestores de saúde. Os usuários foram selecionados pelo critério de utilização regular e vinculação à Estratégia Saúde da Família. Os trabalhadores da saúde compunham equipes no Núcleo de Apoio à Saúde da Família e da equipe da Unidade Básica de Saúde. Os gestores deveriam ser coordenadores gerenciais do programa no município.

A coleta dos dados ocorreu no período de outubro de 2016 a janeiro de 2017, por meio de entrevista semiestruturada ${ }^{(21)}$. Utilizou-se um roteiro contendo questões que pautaram o funcionamento do polo, as potencialidades e fragilidades do programa, bem como a integração do programa na rede de saúde visando à promoção da saúde e prevenção de doenças. O enfoque central das perguntas se relacionou com a efetividade do programa no alcance da qualidade de vida e consolidação das políticas públicas de saúde.

Com indicação da própria equipe de saúde, os usuários foram convidados a participar da presente pesquisa em suas atividades no polo. Houve uma abertura informativa por parte dos gestores em relatar as informações. Os pesquisadores estavam identificados e autorizados previamente para realização dos procedimentos. As entrevistas foram agendadas previamente, gravadas com autorização dos participantes, realizadas em local reservado, com duração de 20 minutos cada e em um clima de cordialidade entre entrevistado e entrevistador.

A interrupção da coleta de dados ocorreu por saturação(22). A amostra, portanto, constou de 21 participantes (15 usuários, três trabalhadores da saúde e três gestores).

A análise dos dados foi pautada na proposição metodológica e analítica fundamentada na hermenêutica crítica(19), cuja apresentação das narrativas compõe os sentidos e significados expressos a partir dos questionamentos e da análise. O processo de análise dos dados envolve três etapas: ordenação dos dados, classificação e análise final. Durante a ordenação dos dados, o material coletado foi remontado para a forma escrita, por meio de transcrições e anotações, consolidados pelo pesquisador. Realizou-se a leitura do material escrito, em forma de narrativas, de maneira exaustiva, organizando-se os dados contemplados.

A classificação dos dados iniciou-se com a leitura flutuante e exaustiva das narrativas, estabelecendo relações com o intuito de formar unidades de sentido a partir das ideias centrais sobre o tema em questão. $A$ análise dos dados empíricos teve como referência as categorias resultantes do cruzamento entre as diferentes informações e a articulação com o referencial teórico ${ }^{(19)}$. Assim, emergiram três categorias temáticas: "A operacionalidade do Programa Academia da Saúde"; "Promoção da saúde e qualidade de vida dos usuários do PAS"; e "Integralidade das ações: articulação com as equipes da ESF e NASF".

Os entrevistados foram informados sobre os objetivos, finalidades e procedimentos da pesquisa, e convidados a participar voluntariamente, concordando por meio de assinatura do Termo de Consentimento Livre e Esclarecido, que segue os princípios éticos em pesquisas envolvendo seres humanos ${ }^{(23)}$. Tendo em vista a necessidade de resguardar as identidades dos participantes, foram atribuídos códigos: para o município (Mun01, Mun02, Mun03); para os usuários (US01...); para os trabalhadores de saúde (TS01...); e para os gestores (GE01...).

O projeto recebeu aprovação pelo Comitê de Ética em Pesquisa da Universidade Regional do Cariri (URCA) sob o Parecer $n^{\circ} .328 .933$.

\section{RESULTADOS E DISCUSSÃO}

Neste espaço serão apresentados e discutidos os resultados encontrados no estudo, agrupados nas seguintes categorias: "A operacionalidade do Programa Academia da Saúde"; "Promoção da saúde e qualidade de vida dos usuários do PAS" e "Integralidade das ações: articulação com as equipes da ESF e NASF", que serão discutidas à luz da literatura. 


\section{A operacionalidade do Programa Academia da Saúde}

Nesta categoria, são apresentados os dados em relação ao funcionamento e organização dos PAS, destacando as facilidades e dificuldades observadas pelos participantes do estudo, bem como as principais ações desenvolvidas.

A operacionalidade é um conceito central quando se discute o PAS para o alcance de resultados quanto à promoção da saúde e impacto na qualidade de vida das pessoas. Uma das principais questões relacionadas à operacionalidade envolve o funcionamento e a organização dos polos. O seu funcionamento deve estar alinhado com a proposta de intervenção. Os resultados, contudo, revelaram divergências em relação aos municípios.

Os usuários do Mun01 consideram que o funcionamento dos polos é bom, acessível para toda a população e destacam como aspectos positivos o fato de ser público/gratuito e ter um profissional conduzindo as atividades. $\mathrm{Na}$ perspectiva do trabalhador da saúde do mesmo município, embora considere que o polo funciona muito bem, ressalta a necessidade de maiores investimentos, uma vez que a demanda tem aumentado em virtude do reconhecimento dos benefícios para a população.

Entre os participantes do Mun02 prevaleceu uma percepção negativa quanto ao funcionamento dos polos. $\mathrm{Na}$ perspectiva de alguns usuários, a infraestrutura e os equipamentos são "precários". Para o trabalhador de saúde, os insumos para o desenvolvimento das ações ainda são insuficientes. Do ponto de vista do gestor, por sua vez, há fragilidades no funcionamento devido à falta de recursos financeiros. Cabe destacar que, nesse município, as atividades do PAS foram paralisadas por um determinado período devido a questões políticas, conforme revelado nas falas dos participantes.

Os usuários do Mun03 definem como bom o funcionamento do polo e destacam que ele tem contribuído para a melhoria da autoestima e qualidade de vida. Embora um dos usuários tenha caracterizado o funcionamento como razoável, não justificou. Na perspectiva do profissional, é regular e promissor, sugerindo que muito ainda pode ser feito para alcançar o ideal proposto para sua estrutura e organização. O gestor do Mun03 não respondeu todas as perguntas, pois estava apenas há dois meses na coordenação do programa, mas relatou reconhecer grande incentivo por parte da gestão para que o polo do programa tivesse um funcionamento efetivo:

"[...] Eu acho assim, que tanto ele agrega o conhecimento das pessoas, a gente faz mais amizades, conhece mais as pessoas, tem mais informações sobre saúde, está sempre havendo eventos de informações como já houve de saúde mental, as campanhas de câncer de mama e essas outras, tudo tem aqui, [...], acontece aqui, como aqui concentra mais pessoas é um meio muito bom pra divulgar as campanhas." (Mun01/Us03)

"O funcionamento eu acho que ainda é um pouco precário, até porque a cidade já está grande e merecia uma coisa maior, um espaço maior, mais dedicado, mas é uma iniciativa bacana, são poucas cidades aqui na região que têm isso. Acho uma iniciativa bacana, mas ainda precisa de um maior olhar do investimento público." (Mun02/Us02)

"[...] considero promissora e regular. [...] então, como se sabe, as pessoas necessitam de programas como esse, que otimizem a promoção e a qualidade de vida dos envolvidos, então, quanto mais participantes, melhor será a saúde da população e menores serão a ingestão medicamentosa [...]." (Mun03/Tb01)

A avaliação dos atores sociais envolvidos na condução e na participação das ações dos polos do PAS também revelaram facilidades e dificuldades nesse processo. As principais facilidades percebidas pelos usuários dos três municípios foram: fácil localização, flexibilidade no horário das atividades, gratuidade e possibilidade de usar os diferentes equipamentos presentes nos polos. As facilidades relatadas pelos profissionais da saúde e gestores foram variadas e enfatizaram a experiência profissional como facilitadora no desenvolvimento das ações de cuidado. Além disso, destacam a flexibilidade em relação aos locais de prática de atividades físicas, bem como o apoio do NASF e da Secretaria de Saúde, sendo a APS responsável pelas ações e planejamentos:

\section{"Ah, porque é pertinho, é seguro [...]." (Mun01/Us03)}

"Como é uma área pública, tem muitas facilidades de você tirar um tempinho e vir praticar algum exercício; como não tem vigia pra você pedir permissão, você chega a hora que quer e sai a hora que quer, isso facilita muito de você frequentar a academia." (Mun02/Us01)

"A facilidade é a questão do trabalho, por ser perto e porque, a cada dia que passa, você se desenvolve mais ainda na academia." (Mun03/Us02)

Em relação às dificuldades, observaram-se algumas especificidades entre os grupos de participantes. Os usuários reconhecem que as limitações pessoais relacionadas às condições de saúde acabam se configurando 
como uma dificuldade para participar das ações do PAS. Outras dificuldades relatadas foram: falta de investimentos, infraestrutura inadequada e ausência de manutenção dos equipamentos:

"A dificuldade, a minha, é por causa de dores que eu já tenho, problemas de dores nas juntas, e eu acordo assim, com dor, e não tenho coragem de vir esquentar aqui." (Mun01/Us03)

"Investimento, só investimento, porque a parte estrutural já tem. Se tivesse investimento pra gente comprar outros materiais, outros equipamentos, assim, seria melhor [...]." (Mun01/Tb01)

"Só que eu acho que deveria ter mais incentivo, tanto do governo como da prefeitura. Deveria ter gestores que auxiliassem mais. Eu acho que está um pouco abandonada." (Mun02/Us05)

"A maior dificuldade é a falta de tempo." (Mun03/Us03)

Estudo transversal ${ }^{(4)}$ relacionado com a efetividade do programa, apontou cerca de $72,8 \%$ dos usuários entrevistados com alto grau de contentamento. Quanto às dificuldades que podem interferir na continuidade da participação dos usuários no PAS, em pesquisa avaliativa( ${ }^{(4)}, 90,6 \%$, deixaram de participar por motivos pessoais e $9,4 \%$, por motivos relacionados ao programa. Entre os motivos pessoais, destacam-se falta de tempo, doença, distância do polo, falta de coragem pessoal em frequentar um programa de atividade física. Por sua vez, os motivos relacionados ao programa foram: horário das aulas, percepção de que o programa é fraco e falta de material para a realização das atividades.

Os resultados quanto às ações desenvolvidas, revelaram que as atividades nos polos estão relacionadas, principalmente, às práticas corporais e atividades físicas. Destacam-se: caminhada; alongamento; exercícios de flexão, abdominal e na barra fixa; corrida; aeróbica; dança e step. Além disso, consideram o espaço da academia como um local de socialização, além de manter contato com a natureza:

"Caminhada, exercício de alongamento, exercício feito com ela [profissional] também, que, três vezes na semana, nós fazemos uns exercícios com ela, que é ótimo também, e a caminhada, que é fundamental. Na segunda, quarta e sexta que nós fazemos os exercícios. Ela começa na faixa de quinze para seis, dez para seis, que é o horário que o pessoal tem mais gente aqui." (Mun01/Us02)

"Eu pratico abdominal, flexão, também dou umas "carreirinhas", umas caminhadas, e o resultado está sendo bom." (Mun02/Us01)

"As principais atividades, que a gente faz são uso da bola, a dança e o step. Eu mesmo aproveito muito em cada um deles [...]." (Mun03/Us02)

Segundo o trabalhador da saúde do Mun02, também são realizadas atividades de educação em saúde, como orientações sobre hábitos de vida saudáveis:

"[...] Questão de alimentação, questão de atividades mesmo que eles poderiam estar fazendo nos dias que a gente não poderia se encontrar [...]." (Mun02/Tb01)

As mesmas ações são realizadas no Mun03:

"[...] além de nós termos o acompanhamento individualizado, como consultas, orientações, nós tivemos ainda atividades coletivas, como palestras, nas quais nós tínhamos orientações diretas dos profissionais do NASF e da ESF." (Mun03/Tb01)

Os resultados do Monitoramento de 2016 do PAS $^{(24)}$ revelam que, nos 1.372 municípios respondentes, 98,6\% informaram que oferecem práticas corporais e atividades físicas, 81,9\% desenvolvem ações de alimentação saudável e $79,2 \%$ realizam ações de educação em saúde. Em $61,7 \%$ há atividades para o enfrentamento ao uso do tabaco e, em $49,3 \%$, para o enfrentamento do uso abusivo de álcool e outras drogas. Em relação às outras atividades (promoção da cultura da paz, abordagem de temas sobre mobilidade segura e desenvolvimento sustentável, ofertas de práticas artísticas e culturais e/ou as práticas integrativas e complementares) foi observado um baixo percentual(24). Esses dados corroboram os resultados de outro estudo ${ }^{(8)} \mathrm{em}$ que prevaleceram as práticas corporais e atividades físicas, associadas a ações de educação em saúde.

\section{Promoção da saúde e qualidade de vida dos usuários do PAS}

Em relação a essa categoria, os usuários dos polos do Programa Academia da Saúde, nos municípios pesquisados, têm percebido um impacto positivo das atividades na melhoria da sua condição de saúde e qualidade 
de vida. Eles atribuem à realização das atividades o controle de doenças crônicas, como hipertensão arterial e diabetes mellitus, a prevenção de doenças, a melhoria de sintomas relacionados a determinadas condições de saúde, a adoção de hábitos saudáveis e o bem-estar:

"Têm muitas pessoas aqui que têm problemas, não é, de artrite, artrose... esses problemas que vão começando, assim, com a idade, e todo mundo se diz melhor depois que começou a frequentar." (Mun01/Us03)

"Ah, muito. Meu problema de pressão, quem tem diabetes, não é, é muita coisa boa. Evita muita coisa, não é, pra quem frequenta." (Mun01/Us04)

"[...] ele vai trazer melhorias na saúde, porque o exercício físico faz reviver a alma, reviver o corpo, malhar, trabalhar, fazer o sangue circular no corpo..." (Mun02/Us02)

"Da melhor forma. As pessoas adquirem hábitos saudáveis, bem-estar, uma autoestima elevada e, assim, o polo, eu acho assim, de grande valia para a população. O pessoal procura muito, é muito bom, muito importante estar praticando exercícios físicos para que possamos ter uma vida saudável." (Mun03/Us04)

A narrativa do gestor do Mun02 corrobora as falas dos usuários sobre a contribuição das ações desenvolvidas nos polos para a promoção da saúde e qualidade de vida da população:

"[...] todo mês a gente verificava e via uma quedazinha de glicemia, uma pressão arterial baixando um pouco, já se sentia um controle de tais situações, e exercício físico é hábito. E se você vai um, dois, três, quatro, cinco dias, se na outra semana você não vai, sente-se falta." (Mun02/Gt01)

Diante dessas falas, ressalta-se que a prática de promoção da saúde e de um estilo de vida saudável deve ser priorizada dentro das ações que são desenvolvidas pelos profissionais que integram os polos do Academia da Saúde.

Os resultados de outra pesquisa( ${ }^{(2)}$ corroboram o PAS como intervenção de promoção da saúde urbana e equidade. Além de oportunizar atividade física à população mais vulnerável, promovendo o direito à saúde na cidade, parece atuar sobre outros desfechos para além do estilo de vida.

A promoção da saúde é uma necessidade urgente para alcançar a saúde e qualidade de vida da população diante de um cenário preocupante, visto que o mundo enfrenta uma tripla carga de doenças, constituída pela agenda inacabada de doenças transmissíveis, novas doenças emergentes e reemergentes, bem como o aumento sem precedentes de doenças crônicas não transmissíveis.

\section{Integralidade das ações: articulação com as equipes da ESF e NASF}

Essa categoria evidencia que as ações desenvolvidas nos polos da Academia da Saúde precisam estar alinhadas com os outros pontos de atenção da rede de saúde de cada município, principalmente com as equipes da ESF e do NASF, com o objetivo de permitir a continuidade do cuidado e a integralidade das ações. Nos municípios pesquisados houve referência dos trabalhadores da saúde e dos gestores quanto à articulação da ESF e NASF com o PAS, tanto na programação de ações integradas (reuniões mensais são programadas para elaboração de um cronograma de atividades) quanto no acompanhamento individual dos usuários a partir do referenciamento, de acordo com suas necessidades de saúde. Embora o trabalhador de saúde do Mun02 não conheça a realidade dos demais programas no município, reconhece a importância de um trabalho conjunto entre ESF, NASF e PAS:

"[...] o NASF e a Estratégia de Saúde da Família, todos apóiam esse programa da Academia da Saúde, todos ajudam. A gente faz inúmeros eventos, tem várias parcerias." (Mun01/Tb01)

"É um trabalho bem articulado. Mensalmente, as equipes se reúnem com a coordenação para planejar e elaborar o cronograma das atividades. [...] Todas as ações desenvolvidas nos dois polos de Academia da Saúde visam atender aos princípios do SUS, dentre eles a integralidade das ações e dos serviços. As atividades coletivas desenvolvidas estão organizadas de acordo com as necessidades identificadas nos serviços de saúde do município." (Mun01/Gt01)

"[...] Nós temos a parceria, no NASF, quanto aos profissionais que atendem, a psicóloga e a nutricionista; na ESF, que são os médicos, técnicos e enfermeiros, que fazem parte da vida e do cuidado em saúde da população." (Mun03/Tb01)

Em um estudo(17) sobre o programa no município do Rio de Janeiro, as ações intersetoriais ocorriam de forma efetiva e as atividades desenvolvidas iam além das práticas de atividade física, o que torna mais eficaz o funcionamento do programa e, consequentemente, leva a um impacto positivo no cotidiano da população atendida. 
Os resultados do Monitoramento 2016 do PAS $^{(24)}$ revelam que apenas $39 \%$ dos polos em funcionamento ( $n=529$ ) recebem apoio de forma regular de profissionais da ESF e $29 \%$ dos polos $(n=392)$ do NASF. Esse apoio refere-se ao planejamento conjunto das atividades, bem como suporte técnico, especializado e/ou pedagógico. Esse percentual é considerado pequeno, uma vez que um dos pontos centrais para o desenvolvimento do PAS é a sua integração com a ESF e o NASF, a fim de garantir a integralidade no cuidado ${ }^{(24)}$, bem como o reconhecimento do usuário nos diferentes pontos da RAS, contribuindo para a continuidade do cuidado.

Entende-se que, para um bom funcionamento e impacto comunitário relevante do PAS, faz-se necessário uma articulação entre gestores e equipe multidisciplinar, em diferentes pontos de atenção da RAS, de modo que os papéis de cada um sejam reconhecidos por todos, estabelecendo uma relação intersetorial, visando manter uma assistência integral prestada ao usuário, família e comunidade.

Embora o atual estudo não tenha um caráter avaliativo, conhecer as percepções dos principais atores envolvidos no PAS (usuários, trabalhadores de saúde e gestores) representa um primeiro olhar nesses municípios, e indica a necessidade de um monitoramento sobre o processo de implementação e avaliação das ações desenvolvidas. Os dados aqui apresentados mostram que existem muitos desafios para o PAS em diferentes contextos. Existe um longo caminho a ser percorrido pelos atores sociais envolvidos na operacionalização do PAS, uma vez que colocar em prática seus objetivos para o fortalecimento de modos de vida saudáveis e da qualidade de vida para indivíduos e coletividades é um processo complexo e processual ${ }^{(26)}$.

A rápida expansão do PAS não tem sido acompanhada de uma avaliação de todo o processo de implementação em diferentes contextos, bem como a compreensão das suas características, tipo de ações, resultados e outros aspectos que compõem essa iniciativa ${ }^{(27)}$. Dessa forma, ressalta-se a contribuição do presente estudo para o planejamento das práticas, para a reavaliação das metas e da necessidade de maiores investimentos, além do fortalecimento do programa e das políticas, dentre elas a de Promoção da Saúde. Devem-se considerar que estratégias de promoção da saúde, como é o caso do PAS, visam impactos de longo prazo, sendo importante definir caminhos para assegurar sua sustentabilidade na agenda local|(8).

Os resultados encontrados sinalizam a relevância de conhecer as percepções dos participantes envolvidos em determinados programas ou ações para o cuidado e promoção da saúde. Além disso, podem servir de incentivo à sua replicação em outros contextos ou áreas de conhecimento.

De modo estratégico, a análise das situações favoráveis e desfavoráveis para operacionalidade do PAS indica caminhos mais resolutivos para municípios que já implantaram ou pretendem dispor do serviço. Sendo assim, os enunciados podem servir de partida situacional para planejamento em outros contextos. Embora limitado à realidade pesquisada e ao foco intersubjetivo das ações, o estudo demonstra uma avaliação participativa das políticas de saúde integradas ao território.

\section{CONSIDERAÇÕES FINAIS}

De maneira geral, nos municípios pesquisados, o estudo permitiu perceber os ganhos que o PAS tem proporcionado, o seu potencial e os caminhos que precisam ser traçados para a sua operacionalidade efetiva. No que se refere ao funcionamento, tem sido considerado bom, apesar de alguns participantes do estudo revelarem as possibilidades de melhoria e aprimoramento dos polos em cada contexto estudado.

As dificuldades compreendidas estão relacionadas, principalmente, aos aspectos estruturais, de materiais e insumos. Em relação às facilidades apresentadas, é possível perceber que a estratégia de implantação dos polos em lugares públicos influencia positivamente na acessibilidade dos usuários, o que corresponde a um resultado buscado e planejado pela equidade promovida nas diretrizes que regulamentam o programa.

Os usuários, os trabalhadores de saúde e os gestores compartilham a mesma percepção em relação à melhoria nas condições de saúde da população atendida nos polos, isto é, de que as atividades oferecidas têm contribuído positivamente no controle de doenças crônicas, como hipertensão arterial e diabetes mellitus. Além disso, associam o programa à qualidade de vida que os usuários têm referido, considerando que essas atividades melhoram a autoestima, revigoram o corpo e a alma.

No que tange ao processo de planejamento e implementação das atividades, considera-se como central, na proposta do PAS, a integralidade e intersetorialidade das ações, principalmente com a ESF e o NASF. Nos municípios pesquisados houve essa articulação com o PAS, tanto na programação de ações integradas quanto no acompanhamento individual dos pacientes, a partir do referenciamento de acordo com suas necessidades de saúde. 
Conhecer as fragilidades do PAS em funcionamento propõe a intervenção dos atores responsáveis, ainda dentro da esfera administrativa municipal, de suma importância para que o seu rendimento desenvolva bons frutos para a saúde e qualidade de vida da população.

Espera-se que este estudo ofereça subsídios para ampliar as ações de promoção à saúde e qualidade de vida nos polos do PAS, promover a integralidade das práticas e dos serviços de saúde, bem como estabelecer novas relações de cuidado entre os usuários.

\section{AGRADECIMENTOS}

Aos participantes do estudo, usuários, gestores e trabalhadores de saúde envolvidos no Programa Academia da Saúde na Macrorregião de saúde do Cariri.

\section{CONFLITOS DE INTERESSE}

Os autores informam que não há conflitos de interesse no estudo realizado.

\section{CONTRIBUIÇÕES}

Laís Barreto de Brito Gonçalves, Rachel Cardoso de Almeida e Tainá Maranhão de Oliveira contribuíram com a concepção da pesquisa; coleta, análise e discussão dos dados; redação do artigo; Maria Augusta Vasconcelos Palácio contribuiu com a análise e discussão dos dados, da redação e revisão crítica do artigo; Antônio Germane Alves Pinto contribuiu com a pesquisa; desenvolvimento da pesquisa; análise e discussão dos dados; redação e revisão crítica do artigo.

\section{FONTES DE FINANCIAMENTO}

FUNCAP/MS/ Secretaria de Saúde do Estado do Ceará/ CNPq

\section{REFERENCIAS}

1. Brasil. Ministério da Saúde. Portaria $n^{\circ}$. 1.707, de 23 de setembro de 2016. Diário Oficial da República Federativa do Brasil, Brasília. 2016.

2. Ministério da Saúde (BR). Academia da Saúde. Brasília: Ministério da Saúde; 2014.

3. Silva RN, Guarda FRB, Hallal PC, Martelli PJL. Avaliabilidade do Programa Academia da Saúde no Município do Recife, Pernambuco, Brasil. Cad Saúde Pública. 2017;33(4):1-16.

4. Hallal PC, Tenório MCM, Tassitano RM, Reis RS, Carvalho YM, Cruz DKA, et al. Avaliação do programa de promoção da atividade física Academia da Cidade de Recife, Pernambuco, Brasil: percepções de usuários e não-usuários. Cad Saúde Pública. 2010;26(1):70-8.

5. Hallal PC, Cordeira K, Knuth AG, Mielke GI, Victora CG. Ten-year trends in total physical activity practice in Brazilian adults: 2002-2012. J Phys Act Health. 2014;11(8):1525-30.

6. Ministério da Saúde (BR). Plano de ações estratégicas para o enfrentamento das doenças crônicas não transmissíveis (DCNT) no Brasil 2011-2020. Brasília: Ministério da Saúde; 2011.

7. Florindo AA, Nakamura PM, Farias JC Jr, Siqueira FV, Reis RS, Cruz DKA, et al. Promoção da atividade física e da alimentação saudável e a saúde da família em municípios com academia da saúde. Rev Bras Educ Fís Esp. 2016;30(4):913-24.

8. Sá GBAR, Dornelles GC, Cruz KG, Amorim RCA, Andrade SSCA, Oliveira TP, et al. O Programa academia da Saúde como estratégia de promoção da saúde e modos de vida saudáveis: cenário nacional de implementação. Cienc Saúde Colet. 2016;21(6):1849-60.

9. Coelho CS, Verdi MIM. Políticas e programas de atividade física: uma crítica à luz da promoção da saúde. Saúde Transform Soc. 2015;6(3):96-108. 
10. Malta DC, Silva MMA, Albuquerque GM, Lima CM, Cavalcante T, Jaime PC, Silva JB Jr. A implementação das prioridades da Política Nacional de Promoção da Saúde, um balanço, 2006 a 2014. Ciênc Saúde Colet. 2014;19(11):4301-11.

11. Ministério da Saúde (BR). Política Nacional de Promoção da Saúde. 3a. ed. Brasília: Ministério da Saúde; 2010.

12. Cavalcanti PB, Lucena CMF. O uso da promoção da saúde e a intersetorialidade: tentativas históricas de integrar as políticas de saúde e educação. Polêm!ca. 2016;16(1):24-41.

13. Mota PHS, Viana ALÁ, Bousquat A. Relações federativas no Programa Academia da Saúde: estudo de dois municípios paulistas. Saúde Debate. 2016;40(108):64-73.

14. Silva RN. Avaliabilidade do programa academia da saúde no Recife: um estudo de caso [dissertação]. Recife: Universidade Federal de Pernambuco; 2015.

15. Lemos EC, Paes IMBS, Abath MB, Brainer MGF, Lima JIAF. Monitoramento do Programa Academia das Cidades e da Saúde: a experiência de Pernambuco. Rev Bras Ativ Fís Saúde. 2015;20(2):203-7.

16. Feitosa WMN, Guarda FRB, Konrad LM, Gonçalves WSF, Martelli PJL, Araújo JLAC Jr. Percepção das usuárias a respeito das ações, melhoria da qualidade de vida e satisfação com o Programa Academia da Cidade. Rev Bras Ativ Fís Saúde. 2016;21(5):461-9.

17. Padilha MA, Oliveira CM, Figueiró AC. Estudo de avaliabilidade do Programa Academia Carioca da Saúde: desafios para a promoção da saúde. Saúde Debate. 2015;39(105):375-86.

18. Guarda F, Silva R, Feitosa W, Santos P Neto, Araújo J Jr. Caracterização das equipes do Programa Academia da Saúde e do seu processo de trabalho. Rev Bras Ativ Fís Saúde. 2015;20(6):638-49.

19. Minayo MCS. O desafio do conhecimento: pesquisa qualitativa em saúde. 14ª . ed. São Paulo: Hucitec; 2014.

20. Ceará. Secretaria de Saúde do Estado. Sala de situação em atenção primária: informações sobre o Programa Academia da Saúde 2012/2013 - Número e tipos de polo [Internet]. 2013 [acesso em 2014 Mar 9]. Disponível em: http://salasituacao.saude.ce.gov.br/salas/index.php/outras-informacoes-georeferenciadas/ atencao-primaria.

21. Fontanella BJB, Luchesi BM, Saidel MGB, Ricas J, Turato ER, Melo DG. Amostragem em pesquisas qualitativas: proposta de procedimentos para constatar saturação teórica. Cad Saúde Pública [Internet]. 2011 [acesso em 2018 Dez 4];27(2):389-94. Disponível em: http://dx.doi.org/10.1590/S0102-311X2011000200020.

22. Manzini EJ. Entrevista semiestruturada: análise de objetivos e de roteiros. $2^{\circ}$ Seminário internacional de pesquisa e estudos qualitativos. A pesquisa qualitativa em debate; 2004; Bauru, São Paulo. Bauru: SIPEQ; 2004. 1 CD.

23. Brasil. Ministério da Saúde. Resolução n. 510, de 7 de abril de 2016, sobre pesquisa envolvendo seres humanos, do Conselho Nacional de Saúde, do Ministério da Saúde. Diário Oficial da República Federativa do Brasil, Brasília. 2016.

24. Ministério da Saúde (BR). Panorama nacional de implementação do Programa Academia da Saúde: monitoramento nacional da gestão do Programa Academia da Saúde: ciclo 2016. Brasília: Ministério da Saúde; 2017.

25. Fernandes AP, Andrade ACS, Costa DAS, Dias MAS, Malta DC, Caiaffa WT. Programa Academias da Saúde e a promoção da atividade física na cidade: a experiência de Belo Horizonte, MG, Brasil. Ciênc Saúde Colet. 2017;22(12):3903-14.

26. Carvalho FFB, Jaime PC. O Programa Academia da Saúde - um estabelecimento de saúde da atenção básica. J Manag Prim Heal Care. 2015;6(1):47-64.

27. Becker LA, Gonçalves PB, Reis RS. Programas de promoção da atividade física no Sistema Único de Saúde brasileiro: revisão sistemática. Rev Bras Ativ Fís Saúde. 2016;21(2):110-22. 
Endereço do primeiro autor:

Laís Barreto de Brito Gonçalves

Universidade Regional do Cariri - URCA

Rua Cel. Antônio Luis, 1161

Bairro: Pimenta

CEP: 63105-000 - Crato - CE - Brasil

E-mail: laizynha1@hotmail.com

Endereço para correspondência:

Antônio Germane Alves Pinto

Universidade Regional do Cariri - URCA

Departamento de Enfermagem

Rua Cel. Antônio Luis, 1161

Bairro: Pimenta

CEP: 63105-000 - Crato - CE - Brasil

E-mail: germanepinto@hotmail.com

Como citar: Gonçalves LBB, Almeida RC, Oliveira TM, Palácio MAV, Pinto AGA. Programa academia da saúde: operacionalidade, ações e integração. Rev Bras Promoç Saúde. 2019;32:8381. 